\title{
A comparative study of evaluate dose related feto-maternal effects of syntocinon during labor
}

\author{
Farhin Radhanpuri*, Jyoti Sharma, Deepak A. Desai
}

SBKS Medical Institute and Research Centre, Pipariya, Waghodia, Vadodara, Gujarat, India

Received: 18 July 2015

Accepted: 14 August 2015

\author{
*Correspondence: \\ Dr. Farhin Radhanpuri, \\ E-mail: farheenradhanpuri@yahoo.com
}

Copyright: (c) the author(s), publisher and licensee Medip Academy. This is an open-access article distributed under the terms of the Creative Commons Attribution Non-Commercial License, which permits unrestricted non-commercial use, distribution, and reproduction in any medium, provided the original work is properly cited.

\begin{abstract}
Background: In times, Active management of Third stage is a gold standard rule, to reduce third stage complications. In the same tradition, we conceived the idea of "Active management of Labor", as a whole, to reduce all the problems, maternal and fetal, to a minimum possible level. With this motto, and aim in mind we devised the protocol of study I present forthwith.

Methods: This study was done in Dhiraj Hospital in Obstetrics and Gynaecology department. The study duration was 1 year. It was a comparative study comprising of patients who came in labor room under unit 3 included in group 1 and under other units included in group 2 and 3. In this study, those patients were augmented who came to labor room. These patients were studied after screening inclusion and exclusion criteria \& its outcome was assessed.

Results: Augmentation-delivery interval in primigravida was 4.5 hours in group 1, in group 2 it was 3 hrs, where as in group 3 active phase delivery interval was 8.1 hours, Augmentation-delivery interval in multigravida was 3.5 hours in group 1, in group 2 it was 3 hrs, where as in group 3 active phase delivery interval was 6 hours. The incidence of spontaneous vaginal delivery was higher in group 1 than in group 2 and 3. There was very less or absent of maternal complications observed in group 1 than in other 2 groups. Incidence of NICU admissions was only $1 \%$ in group 1 , whereas in other groups it was higher. There was no neonatal mortality noted in either group.

Conclusions: There is significant reduction in the duration of labor by augmenting labor with slow low regulated dose of syntocinon drip, thus reducing the maternal exhaustion and morbidity due to prolonged labor. There is significant reduction in the operative interference like LSCS, vacuum and forceps delivery, thus reducing maternal morbidity associated with operative interference and anesthesia. It also reduces the cost of medical services. The incidence of fetal distress and LSCS for the same does not increase in the augmentation group, indicating that syntocinon can be safely used for the augmentation. At this time, much attention in the field of obstetrics is focused on attempting to reduce the rate of cesarean section, not only to reduce maternal morbidity, but to lower the cost of medical care. Our finding is that syntocinon administration can significantly reduce the cesarean section rate.
\end{abstract}

Keywords: Augmentation, Syntocinon

\section{INTRODUCTION}

Present study was done to get maximum benefits of syntocinon augmentation in terms of successful vaginal delivery, without any fetal or maternal morbidity. Concomitant reduction in the duration of labor, turned- out to be a bonus. ${ }^{1}$ In spite of efforts, cesarean section may become necessary in course of labor due to a variety of reasons, after excluding pelvic causes; low dose infusion can still be a helpful mode of management. ${ }^{2}$ 


\section{METHODS}

\section{Study design}

In this study, those patients were included who came to labor room. These patients were studied after screening inclusion and exclusion criteria \& its outcome was assessed.

\section{Inclusion criteria}

Patient in active stage of labor, with all normal vital parameters, Gestational age >37 weeks, Vertex presentation.

\section{Exclusion criteria}

Grand multipara, previous c.s., malpresentation, CPD, obstructed labor.

\section{RESULTS}

The present study was conducted over a period of 1 year in labor rooms of Dhiraj hospital, Pipariya. This study evaluates the efficacy of augmentation of labor with, slow low dose syntocinon infusion versus group which includes no induction or augmentation of labor and Groups, wherein augmentation done with high dose syntocinon. ${ }^{3} 90$ patients were included in the study, each group includes 30 patients. It is a comparative study.

Group 1: Augmentation with 1 unit syntocinon

Group 2: Augmentation with $2.5 \& 5$ unit syntocinon

Group 3: No augmentation

Table 1: Distribution according to registration.

\begin{tabular}{|lllllll|} 
& \multicolumn{2}{c}{ Group 1 } & \multicolumn{2}{c}{ Group 2 } & \multicolumn{2}{c|}{ Group 3 } \\
Registration & Booked & Un-Booked & Booked & Un-Booked & Booked & Un-Booked \\
\hline No. Of Patients & 20 & 10 & 21 & 9 & 20 & 10 \\
\hline Percentage & $66.6 \%$ & $33.3 \%$ & $70 \%$ & $30 \%$ & $66.6 \%$ & $33.3 \%$ \\
\hline
\end{tabular}

There was no significant difference between booked and un-booked patients in comparative groups.

Table 2: Distribution according to age group.

\begin{tabular}{|lllllll|} 
& \multicolumn{2}{c}{ Group 1 } & \multicolumn{2}{c}{ Group 2 } & \multicolumn{2}{c|}{ Group 3 } \\
Age (Years) & $21-30$ & $>30$ & $21-30$ & $>30$ & $21-30$ & $>30$ \\
\hline No of Patients & 22 & 8 & 20 & 10 & 20 & 10 \\
\hline Percentage & $73.3 \%$ & $26.6 \%$ & $66.6 \%$ & $33.3 \%$ & $66.3 \%$ & $33.3 \%$ \\
\hline Mean \pm SD & $24 \pm 2.36$ & $32 \pm 1.06$ & $23.78 \pm 2.25$ & $31 \pm 0.70$ & $23.78 \pm 2.25$ & $31 \pm 0.70$ \\
\hline
\end{tabular}

This table shows that there were no significant changes pertaining to the age group in 3 groups.

Table 3: Distribution according to parity.

\begin{tabular}{|lllllll|}
\hline & \multicolumn{2}{c}{ Group 1 } & \multicolumn{2}{c}{ Group 2 } & \multicolumn{2}{c|}{ Group 3 } \\
PARITY & $\begin{array}{l}\text { No of } \\
\text { Pts }\end{array}$ & Percentage & $\begin{array}{l}\text { No of } \\
\text { Pts }\end{array}$ & Percentage & $\begin{array}{l}\text { No of } \\
\text { Pts }\end{array}$ & Percentage \\
\hline PRIMI'S & 23 & $76.6 \%$ & 20 & $66.6 \%$ & 22 & $73.3 \%$ \\
\hline MULTI'S & 7 & $23.3 \%$ & 10 & $33.3 \%$ & 8 & $26.6 \%$ \\
\hline
\end{tabular}

The number of primigravida and multigravida in each group, were kept almost equal, so as to authenticate the result on an even scale.

The gestational age was consciously studied at almost same duration, so as to achieve a result, with a base of clinical reliability in each group.

We did not include patients with height $<150 \mathrm{~cm}$, obese patients were also excluded. In this study the height and weight of the patients included in both the groups were identical. The median interval from the start of augmentation to vaginal delivery was $4.5 \mathrm{hrs}$ in primi's, and $3.5 \mathrm{hrs}$ in muti's, in the group 1. In group 3, control group, active phase delivery interval was $8.1 \mathrm{hrs}$ in primi's and 6 hrs in multi's. This suggests, that there is almost $3.5 \mathrm{hrs}$ difference in primi's and p-value of this data is 0.049 , and $2.5 \mathrm{hrs}$ in multi's- $\mathrm{p}$ value for this data is 0.016 (statistically significant), which matters a lot for a women in labor. In our study, we could manage to decrease this duration appreciably, to alleviate the dread and apprehension, with augmentation of labor with low dose controlled input syntocinon drip. ${ }^{4}$ In group 2 augmentation delivery interval in primi was $4 \mathrm{hrs}$ and in multi it was $3 \mathrm{hrs}$, only half an hour difference from group 1. With the said advantage, there is a chunk of disadvantages, as well. None of them was severe enough to add to the morbidity, or add any mortality, they did occur in few patients, and made its presence felt. In the conducted study, the occurrence of-- tachysystole was 
$0.3 \%$ in the group $1,1 \%$ in the group 2 and $0.6 \%$ in group 3 . Whereas the occurrence of hyperstimulation was $0.3 \%$ in group $1,1.2 \%$ in group 2 and $0.6 \%$ in group 3 . Occurrence of Hypertonus was $0 \%$ in group 1 and 3 and $1 \%$ in group 2 . When tachysystole or hyperstimulation occurred, the syntocinon was stopped, and oxygen and hydration with lactated ringer's solution were immediately given in both the groups. In group 1 occurrence of perineal tear was $1.2 \%$ and cervical tear was $0 \%$, in group 2 perineal tear was $3 \%$ and cervical tear was $0.9 \%$ and in group 3 it was $2.4 \%$ and $0.6 \%$ respectively. Hyperstimulation cases were taken up for emergency cesarean delivery in view of non-reassuring heart rate. In the rest of the women, in whom tachysystole was observed, delivered vaginally, with the fetal heart rate pattern returning to normal, within 5-10 minutes after omitting drip. This data suggestive of very less or absent maternal complications were observed, in the group 1 than in other 2 groups. ${ }^{5}$

Table 4: Distribution gestational age according to parity.

\begin{tabular}{|lllllll|} 
& \multicolumn{2}{c}{ Group 1 } & \multicolumn{2}{c}{ Group 2 } & \multicolumn{2}{c|}{ Group 3 } \\
Parity & Primi's & Multi's & Primi's & Multi's & Primi's & Multi's \\
\hline Gestational age (wks) & 38.4 & 38.6 & 38.5 & 38.3 & 38.6 & 38.3 \\
\hline Mean \pm SD & $38.4 \pm 0.86$ & $38.6 \pm 0.98$ & $38.5 \pm 1.02$ & $38.3 \pm 1.06$ & $38.6 \pm 0.98$ & $38.3 \pm 1.06$ \\
\hline
\end{tabular}

Table 5: Average height and weight.

\begin{tabular}{|lllllll|}
\hline & Group 1 & \multicolumn{3}{c}{ Group 2 } & \multicolumn{2}{c|}{ Group 3 } \\
\hline Parameters & Height & Weight & Height & Weight & Height & Weight \\
\hline Mean & $154 \mathrm{cms}$ & $59 \mathrm{kgs}$ & $153 \mathrm{cms}$ & $58 \mathrm{kgs}$ & $153 \mathrm{cms}$ & $59 \mathrm{kgs}$ \\
\hline Mean \pm SD & $154 \pm 4.58$ & $59 \pm 5.70$ & $153 \pm 3.52$ & $58 \pm 3.66$ & $153 \pm 3.52$ & $59 \pm 5.70$ \\
\hline
\end{tabular}

Table 6: Augmentation - delivery interval.

\begin{tabular}{|c|c|c|c|c|c|c|}
\hline \multirow[b]{3}{*}{ Parity } & \multicolumn{2}{|c|}{ Group 1} & \multicolumn{2}{|c|}{ Group 2} & \multicolumn{2}{|c|}{ Group 3} \\
\hline & \multicolumn{2}{|c|}{$\begin{array}{l}\text { Augmentation- } \\
\text { Delivery interval }\end{array}$} & \multicolumn{2}{|c|}{$\begin{array}{l}\text { Augmentation- } \\
\text { Delivery interval }\end{array}$} & \multicolumn{2}{|c|}{$\begin{array}{l}\text { Active phase- Delivery } \\
\text { interval }\end{array}$} \\
\hline & PRIMI'S & MULTI'S & PRIMI'S & MULTI'S & PRIMI'S & MULTI'S \\
\hline Mean Duration & $\begin{array}{l}4.5 \mathrm{hrs} \\
(4 \mathrm{hrs} 50 \\
\text { mins })\end{array}$ & $\begin{array}{l}3.5 \mathrm{hrs} \\
(3 \mathrm{hrs} 50 \\
\text { mins })\end{array}$ & $4 \mathrm{hrs}$ & $3 \mathrm{hrs}$ & $\begin{array}{l}8.1 \mathrm{hrs} \\
(8 \mathrm{hrs} 10 \\
\text { min) }\end{array}$ & $6 \mathrm{hrs}$ \\
\hline Mean \pm SD & $4.5 \pm 0.44$ & $3.5 \pm 0.59$ & $4 \pm 0.41$ & $3 \pm 0.32$ & $8.1 \pm 0.63$ & $6 \pm 0.82$ \\
\hline
\end{tabular}

Table 7: Maternal complications in both the groups.

\begin{tabular}{|llll|}
\hline Complications & Group 1 & Group 2 & Group 3 \\
\hline Tachysystole & $0.3 \%$ & $1 \%$ & $0.6 \%$ \\
\hline Hypertonus & $0 \%$ & $1 \%$ & $0 \%$ \\
\hline Hyper stimulation & $0.3 \%$ & $1.2 \%$ & $0.6 \%$ \\
\hline Perineal tear & $1.2 \%$ & $3 \%$ & $2.4 \%$ \\
\hline Cervical tear & $0 \%$ & $0.9 \%$ & $0.6 \%$ \\
\hline
\end{tabular}


Table 8: Mode of delivery.

\begin{tabular}{|lllll|}
\hline Mode of delivery & Group $\mathbf{1}$ & Group 2 & Group 3 & P-VALUE \\
\hline FTVD & 28 & 24 & 25 & 0.0212 \\
\hline Instrumentation & 1 & 2 & 2 & 0.3605 \\
\hline LSCS & 1 & 4 & 3 & 0.040 \\
\hline
\end{tabular}

In this study $93.3 \% \%$ achieved a vaginal delivery and $3.3 \%$ underwent cesarean delivery and instrumentation in $3.3 \%$ in group 1 . In the group $2,80 \%$ had vaginal delivery and $13.3 \%$ underwent cesarean delivery, $6.6 \%$ instrumental delivery in group 3 it was $83.3 \%, 6.6 \%$, $10 \%$ respectively, which is suggestive of less operative morbidity in group 1 .

Table 9: Indication for cesarean section.

\begin{tabular}{|l|lll|}
\hline Indication of cesarean section & Group 1 & Group 2 & Group 3 \\
\hline NPOL & 1 & 0 & 2 \\
\hline FD & 0 & 3 & 1 \\
\hline Thick MSL & 0 & 1 & 0 \\
\hline
\end{tabular}

In the conducted study, 1 pt underwent cesarean delivery in group 1 for NPOL. 4 pts underwent cesarean delivery in the group 2, of which 3 were done due to fetal distress and 1 were done due to thick MSL, in group 3, 3 pts underwent cesarean delivery, 2 for NPOL and 1 for FD. Suggestive of number of cesarean sections due to FD were more in group 2 , and due to NPOL in group 3 . In the conducted study, 1 pt underwent cesarean delivery in group 1 for NPOL. 4 pts underwent cesarean delivery in the group 2, of which 3 were done due to fetal distress and 1 were done due to thick MSL, in group 3, 3 pts underwent cesarean delivery, 2 for NPOL and 1 for FD. Suggestive of number of cesarean sections due to FD were more in group 2, and due to NPOL in group 3.

Table 10: Neonatal outcome.

\begin{tabular}{|llll|}
\hline Neonatal outcome & Group 1 & Group 2 & Group 3 \\
\hline $\begin{array}{l}\text { Apgar score }<7 \\
\text { 1' min }\end{array}$ & 1 & 2 & 1 \\
\hline 5' $\mathrm{min}$ & - & 1 & 1 \\
\hline 10' $\mathrm{min}$ & - & - & \\
\hline Admission to NICU & 1 & 3 & 2 \\
\hline
\end{tabular}

In group 1 Apgar score $<7$ at 1 min was found in 1 patient, was admitted in NICU for mild to moderate birth asphyxia (a case with hyper stimulation) and in group 2 Apgar score $<7$ at $1 \mathrm{~min}$ found in 2 patients and in 1 patient at $5 \mathrm{~min}$, all 3 were admitted in NICU for birth asphyxia and in group 3,2 pts were admitted in NICU for birth asphyxia. There was no neonatal mortality in any group. In group 2, 2 cases of severe birth asphyxia were admitted in NICU for 6 days, which suggests number of NICU admission in group 1 is very less than in other two groups. In times, Active management of Third stage is a gold standard rule, to reduce third stage complications. In the same tradition, we conceived the idea of "Active management of Labor", as a whole, to reduce all the problems, maternal and fetal, to a minimum possible level. Concomitant reduction in the duration of labor, turned out to be a bonus.

\section{DISCUSSION}

This result could be achieved due low dose, slow, well regulated drip. Syntocinon augmenting well-coordinated, rhythmic, efficient uterine contractions, coming at regular intervals. This gradually increased, in frequency and intensity. This pattern tuned and trained the uterine musculature to contract and retracts in well-coordinated manner, without being fatigued. The gradual increase in intra-uterine pressure, adjusted well, without hampering the circulation in sinuses. This averted the intrauterine fetal hypoxia, and so, distress, which commonly occurs, when the contractions are in-co-ordinated, hypertonic and irregular. ${ }^{6}$

This later state of contractions, increase the intra uterine pressure erratically, hampers the circulation through sinuses, resulting in fetal hypoxia and distress. The uterine musculature also gets fatigued with hypertonic contractions, during first two stages of labor. This ultimately results in uterine atony, when it is most undesirable, in $3^{\text {rd }}$ stage, causing P.P.H. This result is 
sometimes found in cases, when labor is allowed to pursue an absolutely natural course of progress, when contractions can become ineffective, erratic and hypertonic due to pacemaker over activity. ${ }^{7}$ These undesirable results could be averted by slow, low dose, regulated infusion.

\section{CONCLUSION}

"Giving birth should be your greatest achievement, not your greatest fear." - Jane Weidman

"It is essential that we want to bring about an easy labor, without risking injury, to the mother or the child; we must go further. We must understand that childbirth is fundamentally a spiritual, as well as a physical, achievement. The birth of a child is the ultimate perfection of human love."

"It seems that many health professionals involved in antenatal care have not realized that one of their roles should be to protect the emotional state of pregnant women." - Michel Odent

In our study, we could manage to decrease the total duration appreciably, to alleviate the dread and apprehension, with augmentation of labor with low dose controlled input of syntocinon drip. Dying in childbirth is something that's not new; it's been going on for ages, and so it's not something that people focus on; it's not something that gets funded for and it's exactly for that reason that we are losing mothers all the time and we have kids with no mother. This is an epitome of callosity, for a mother to be. We intended and aimed to eliminate this callosity.

"There is a secret in our culture, and it's not that birth is painful. It's that women are strong." - Laura Stavoe

In our study, we could also manage to reduce the operative morbidity and mortality, and tried to restrict the morbidity to very minimal, or avert mortal complications, with help of controlled, slow, low dose infusion with syntocinon, which almost mimics natural or physiological release of syntocinon in labor. In women, undergoing the process of labor, there is a physiological pulsatile release of oxytocin which brings about the natural expulsion of baby and the placenta. By supplementing the minimum possible dose of syntocinon for women in labor, there occurs, an addition, of expulsive forces, to the already existing physiological levels, that can help augment the labor, or substitute for the deficient levels, which can regularize the contractions, ultimately reduce labor duration. Side effects are nullified, with more benefits, like reduced maternal operative morbidity, reduced medical costs, and good maternal and neonatal outcome. $^{8,9}$

"Childbirth is more admirable than conquest, more amazing than self-defence and as courageous as either one".

"A woman in labor is at her most vulnerable, and yet her most powerful, self at the same time. Any woman, who has birthed unhindered, understands that, she is stronger than all know".

\section{Funding: No funding sources}

Conflict of interest: None declared

Ethical approval: The study was approved by the Institutional Ethics Committee

\section{REFERENCES}

1. Frigoletto FD, Lieberman E, Lang JM, et al. A clinical trial of active management of labor. $\mathrm{N}$ Engl $\mathrm{J}$ Med. 1995;333:745-50.

2. Rogers R, Gelson GJ, Miller AC, et al. Active management of labor: Does it make a difference? Am J Obstet Gynecol. 1997;177:599-605.

3. Shyken JM, Roy H. Petrie. Oxytocin to augment labor. Clinical Obstetrics and Gynecology. 1995;38 (2):232-45.

4. "Induction and augmentation of labor". ACOG Technical Bulletin, 157 Washington DC, ACOG 1991.

5. David C. Merril, Stephan K. Use of oxytocin for induction and augmentation of labor. 1995;15(18).

6. S Arulkumaran. Management of labor. Chapter 2 RCOG guidelines for induction of labor 2001.

7. Obstetrics \& Gynecology: Active-Phase Labor Arrest: Oxytocin Augmentation for at Least 4 Hours Rouse, Dwight J, Owen, John, Hauth, John C. 1999;93:323-328.

8. Obstetrics \& Gynecology Randomized, DoubleMasked Comparison of Oxytocin Dosage in Induction and Augmentation of Labor Merrill, David C, Zlatnik, Frank J. 1999;94:455-463

9. Obstetrics \& Gynecology: The Effect of Early Oxytocin Augmentation in Labor: A Meta-Analysis Wei, Shu-Qin, Luo, Zhong-Cheng, Xu, Hairong, Fraser, William D. 2009;114:641-649.

Cite this article as: Radhanpuri F, Sharma J, Desai DA. A comparative Study to evaluate dose related feto-maternal effects of syntocinon during labor. Int J Reprod Contracept Obstet Gynecol 2015;4:1344-8. 